\title{
Application of Nano Technique in Measuring Supersonic/Hypersonic Flow
}

\author{
Chen Zhi, YI Shihe, Zhu Yangzhu, Zhang Qinghu, Wu Yu \\ College of Aerospace Science and Engineering, National University of Defense Technology, Changsha, China \\ Email: gfkdchenzhi@163.com
}

Received 2012

\begin{abstract}
Turbulence, universally exist in nature and human activities, is a kind of three-dimensional, irregular, unsteady flow. Ever since $19^{\text {th }}$ century when people started to investigated turbulent flow technically, they have never dropped the potent and intuitionistic experimental method. Recently, with the development of aviation and aerospace industry, especially with the increase desire of supersonic and hypersonic flight, the mechanism of high speed and compressible flow has become hot topic of fluid research, resulting in development of measurement method and technique. When encountering compressible high flow, traditional techniques, such as schilieren, shadow and interference, cannot measure fine flow structures. Fortunately, multiple-discipline integration of nano technique, laser technique and imaging technique provides a new design for fluid measurement Nano-tracer planar laser scattering (NPLS) is a new flow visualization technique, which was developed by the authors' group in 2005, and it can visualize time correctional flow structure in a cross-section of instantaneous 3D supersonic flow at high spatiotemporal resolution. Many studies have demonstrated that NPLS is a powerful tool to study supersonic turbulence.
\end{abstract}

Keywords: Nano Trace; NPLS; Supersonic/Hypersonic; Flow Visualization and Measurement

\section{Introduction}

For the complexity and instability of the supersonic turbulence, measuring its fine structures becomes very difficult. The existing experimental methods have their own shortages for supersonic turbulence study, such as Schlieren, filtered Rayleigh scattering (FRS), and planar laser induced fluorescence (PLIF), and the main reasons are their low resolution or signal noise ratio (SNR) [1]. NPLS, as a newly developed flow visualization technique by the authors' group, can visualize time correctional flow structures in a cross-section of instantaneous 3D supersonic flow at high spatiotemporal resolution [2].

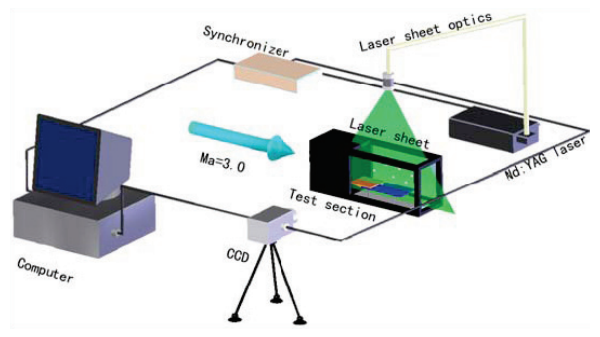

Fig. 1 NPLS Testing System

As shown in Fig 1, it was the NPLS testing system used in this paper. A dual-cavity Nd: YAG pulsed laser was used as its light source, which emitted two laser beams of pulse width of 6 ns according to the scheduling set by the synchronizer. A light sheet of less than $1 \mathrm{~mm}$ thick illuminated the flow field of interest. Owning to the excellent the following ability, nano-particles with nominal diameter of $18 \mathrm{~nm}$ could catch the complicated structures in supersonic flow field and scattered laser light effectively to generate high SNR images. The recording system was an interline transfer double-exposure CCD whose resolution was $2 \mathrm{~K} \times 2 \mathrm{~K}$, and the shortest interval of double-exposure was $0.2 \mu \mathrm{s}$. The synchronizer, whose accuracy was $250 \mathrm{ps}$, can adjust the time of laser emitting and $\mathrm{CCD}$ exposure according to the signal of computer to make sure that the two laser beams were exposed in the frames of dual-exposure respectively. The computer's charges were setting parameters of the synchronizer, storing and processing images. Fig.2 shows a typical supersonic flow image via NPLS.

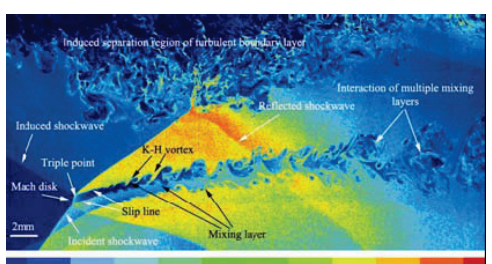

Fig. 2 Typical supersonic flow measured by NPLS 


\section{Performance of NPLS}

\subsection{Visualization of supersonic flow}

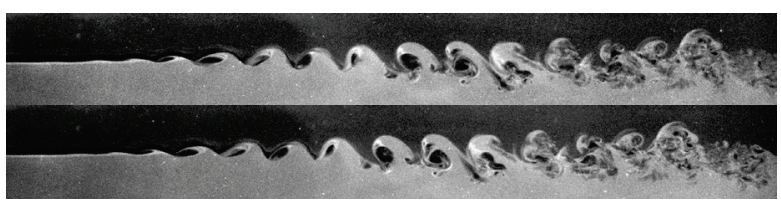

(a) $\mathrm{Mc}=0.24, \Delta \mathrm{t}=5 \mu \mathrm{s}$

In the past several years, spatiotemporal characteristic of supersonic mixing layers with convective Mach number $0.12,0.21,0.24,0.32,0.50$ and 0.60 were studied by the authors. And the results revealed Kelvin-Helmholtz instable vortexes in the flow field, and its spatial features and temporal evolution can be yielded from NPLS images, which are shown in Fig. 3. We also studied its spanwise structure, and found the intriguing vortexes due to the secondary instability, which are shown in Fig. 4 and Fig. 5.

The NPLS technique has been widely used to study other important problems of supersonic mixing layer, including the velocity field of the transition process ${ }^{[8]}$, the turbulent structure with unmatched pressure ${ }^{[6]}$, the fractal characteristics of the mixing interface ${ }^{[9]}$, the multiresolution analysis of the density field ${ }^{[17]}$.

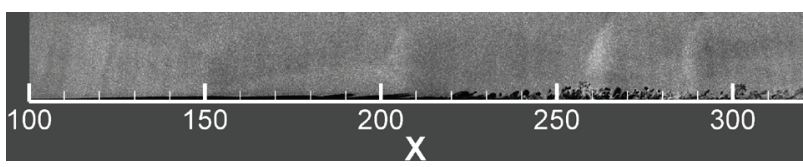

Fig. 8 NPLS image of supersonic boundary layer in $\mathrm{Ma}=3$ flow.

As shown in Fig. 8 is a NPLS image of flat plate boundary layer in vertical plane in $\mathrm{Ma}=3$ flow, and the flow region is $100-320 \mathrm{~mm}$ from the leading edge of plate. Fig. 8 displays the whole transition process of the boundary layer. Until $180 \mathrm{~mm}$ distant from the leading edge, the flow is still laminar. Then follows transition, and flow becomes fully developed from $\mathrm{X}=250$.

\subsection{Visualization of hypersonic flow}

The following ability, scattering characteristic and reuniting of nano particles are the key points under the consideration of NPLS. The distribution of nano particles can reveal the exact flow field structures as long as its following ability meets the flows. However, when tested flow accelerates to hypersonic, the following ability of nano particles would be challenged again. And using directly nano particles of smaller scale sometimes cannot help, due to reuniting. Shown in Fig. 3, it is a hypersonic flow structure measured by NPLS. By now, investigations on separating and scattering nano particles have to be kept going on, in order to improve NPLS technique and make it more applied in hypersonic flow measure- ments.

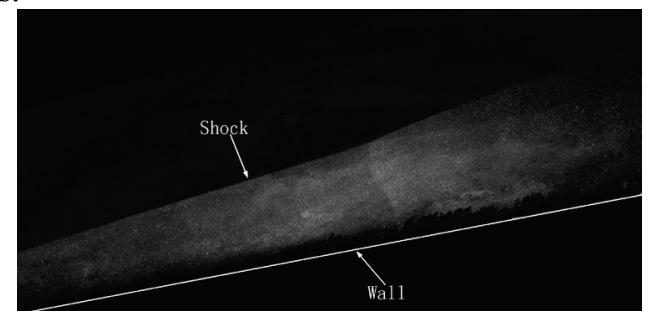

Fig. 3 Hypersonic flow structure measured by NPLS

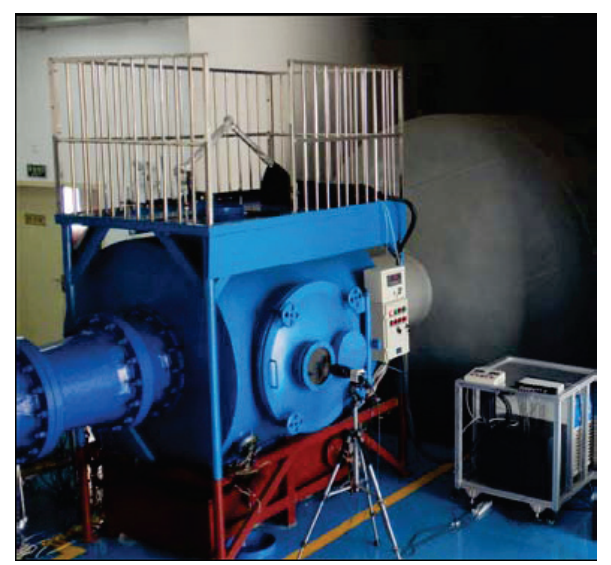

Fig.1 KD-01 free-piston shock tunnel

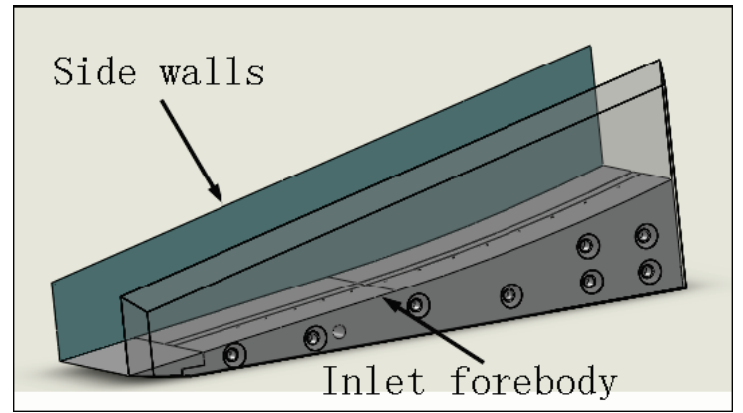

Fig. 2 Schematic diagram of the model

\section{Development and Challenge of NPLS}

\section{Conclusion}

\section{Acknowledgements}

Project supported by National Basic Research Program of China (Grant Nos. 2009 CB724100), National Natural Science Foundation of China (Grant Nos. 11172326) and innovation fund program for standout graduate students of NUDT (Grant Nos. B120103).

\section{REFERENCES}

[1] ZHAO Y X, Yi S H, TIAN L F, et al. Supersonic Flow imaging via nanoparticles. [J]. Science China Ser E- Tech Sci, 2009, 52(12): 3640-3648.

[2] ZHAO Y X, YI S H, TIAN L F, et al. The quantification- 
al measurement of supersonic mixing layer growth rate [J]. Journal of experiments in Fluid Mechanics, 2009, 23(3): 100-103.

[3] YI S H, ZHAO Y X, TIAN L F, et al. Recent advances of experimental study of supersonic mixing layer transition [J]. ACTA AERODYNAMICA SINICA, 2009, 27: 114-119.

[4] TIAN L F, YI S H, ZHAO Y X, et al. Flow visualization of supersonic flow around a concave optical bow cap model [J]. Journal of experiments in Fluid Mechanics, 2009, 23(1): 15-17.

[5] ZHAO Y X, TIAN L F, YI S H, et al. Experimental study of flow structure in pressure unmatched mixing layer [J]. Journal of experiments in Fluid Mechanics, 2007, 21(3): 14-17.

[6] ZHAO Y X, YI S H, HE L, et al. The experimental research of shocklet in supersonic turbulent mix layer [J]. Journal of National University of Defense Technology, 2007, 29(1): 12-15.

[7] ZHAO Y X, YI S H, HE L, et al. The experimental study of interaction between shock wave and turbulence [J]. Chinese Science Bulletin, 2007, 52(10): 1297-1301.

[8] ZHAO Y X, YI S H, HE L, et al. The fractal measurement of experimental images of supersonic turbulent mixing layer $[\mathrm{J}]$. Sci China Ser G-Phys Mech Astron, 2008, 51(8): 1134-1143.

[9] Zhao Y X. Experimental investigation of spatiotemporal structures of supersonic mixing layer [D]. $\mathrm{PhD}$ Thesis, National University of Defense, 2008.

[10] Zhao Y X, Yi S H, TIAN L F, et al. An experimental study of aero-optical aberration and dithering of supersonic mixing layer via BOS [J]. SCIENCE CHINA: Physics, Mechanics \& Astronomy, 2009, 53(1): 81-93.

[11] TIAN L F, YI S H, ZHAO Y X, et al. Study of density field measurement based on NPLS technique in supersonic flow [J]. Sci China Ser G, 2009, 52(9):1357-1363.

[12] ZHAO Y X, YI S H, TIAN L F, et al. Multiresolution analysis of density fluctuation in supersonic mixing layer [J]. Sci China Tech Sci, 2010, 53(2): 584-591.

[13] YI S H, TIAN L F, ZHAO Y X, et al. Aero-optical aberration measuring method based on NPLS and its application [J]. Chinese Sci Bull, 2010, 55(31): 3545-3549.

[14] TIAN L F, YI S H, ZHAO Y X, et al. PIV study of supersonic flow around an optical bow cap [J]. Journal of experiments in Fluid Mechanics, 2010, 24(1): 26-29.

[15] CHEN Zhi, YI ShiHe, HE Lin, TIAN LiFeng \& ZHU YangZhu. An experimental study on fine structures of supersonic laminar/turbulent flow over a backward-facing step based on NPLS [J]. Chinese Science Bulletin, 2012, 57(6):584-590. 000299924600005(SCI)/1.087

[16] Z. Chen, S.H.Yi, L.F.Tian, L. He \& Y.Z.Zhu. Flow visualization of supersonic laminar flow over a backward-facing step via NPLS [J]. Shock Waves, Published online: 18 May 2012. (DOI 10.1007/s00193-012-0378-7) 